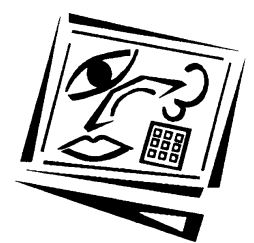

\title{
Developing the clinical psychomotor skills of musculoskeletal sonography using a multimedia DVD: A pilot study
}

\begin{abstract}
Kerry Thoirs and Jane Coffee University of South Australia

Sonographers are medical or non-medical health professionals in the radiology field who skilfully manipulate ultrasound equipment to produce images that are used to diagnose medical conditions and abnormalities. This technique is also becoming popular amongst the wider community in other medical specialities and allied health professionals, due to decreasing costs and automation. This paper reports the findings of a pilot trial on the use of a specifically designed audiovisual tool to teach medical sonography students psychomotor skills to perform sonography of specific musculoskeletal structures of the ankle. A competency testing instrument was developed using the Dawson's psychomotor categories as a framework, which identified positive change in competency in several scanned structures following this intervention. Integrating a conceptual learning framework for psychomotor skills could be useful to guide the development of sonography training programs and assist educators to structure incremental teaching and assessment activities, enabling a more consistent approach to developing psychomotor skills. Alterations in instructional design using this technology may relieve some of the burden of clinical teaching in the workplace.
\end{abstract}

\section{Introduction}

Sonographers are medical or non-medical health professionals in the radiology field who skilfully manipulate ultrasound equipment to produce images that are used to diagnose medical conditions and abnormalities. This technique has also become popular amongst specialists in cardiology, vascular medicine, ophthalmology, obstetrics and gynaecology, and rheumatology. Allied health professionals such as physiotherapists and podiatrists are also looking to utilise and adapt this technology as it becomes more accessible through reduced cost, and increased automation.

The development of psychomotor skills is a primary learning outcome in almost all health practitioner education. Teaching institutions tend to focus on developing learning frameworks around the cognitive and affective domains for health professionals, and leave the development of psychomotor skills to clinical sites where students are provided with supervised practice opportunities. The success of this approach relies on the skill of clinical supervisors to meet and understand the learning needs of their students while also managing the care of their clients or patients. Clinicians with this dual role are experiencing pressure with increasing clinical 
workloads and the increasing number of students being trained in an attempt to provide a workforce that has the capacity to meet future demands on the health service (KPMG, 2009). These conflicting influences potentially impact negatively on the students learning experience to develop clinical skills. It is therefore timely to reconsider the instructional design for the teaching of psychomotor skills for health professionals to maximise the efficiency and quality of the learning experience in supervised practice.

While Bloom's (1956) taxonomy of educational objectives has been a cornerstone for analysing and thinking about the goals of educational activities in the cognitive and affective domains, the original taxonomy did not develop the psychomotor domain. Other authors have addressed this gap by developing frameworks for analysing the learning and acquisition of skills in the psychomotor domain (Dave, 1967; Harrow, 1972; Simpson, 1972). These frameworks broadly categorise four learning stages; observation, imitation, practice, and habit. Dawson (1998) more recently extended Bloom's psychomotor taxonomy, describing four stages, being observation, refinement, consolidation and mastery (Figure 1). Three of these stages, refinement, consolidation and mastery provide opportunities for assessment. To reach these levels within the framework, students commence with an exact preview of the expected outcomes of their learning (observation), are given the opportunity to practise the correct technique (trial) and practise minor adjustments to their technique to achieve consistency (repetition). Students may use observation, trial and repetition between any of the assessable stages to assist their learning. Consolidation occurs when students can replicate a task correctly despite the lapse of time. Mastery is the highest stage requiring the guidance and supervision in an employment setting and reflects the development of the skill to that of a highly skilled practitioner. These stages can be applied in the development of sonography scanning techniques.

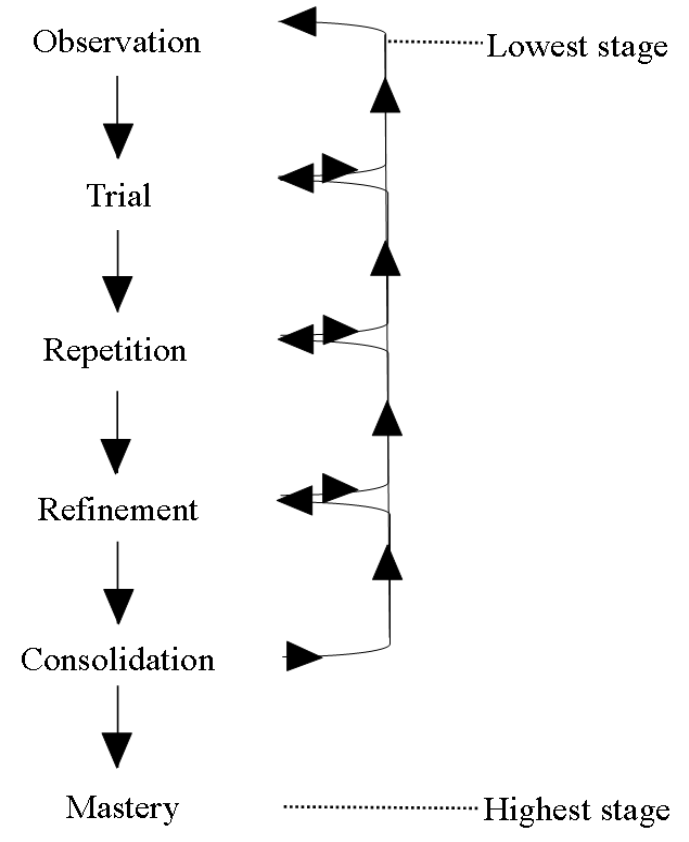

Figure 1: Dawson's (1998) psychomotor learning stages 
Sonographers develop sophisticated skills layered on knowledge of normal and abnormal three-dimensional anatomy together with knowledge of the physics of how sound interacts with human tissue. The sonographer develops an eye-hand neural loop where the hand takes cues from real time images on a monitor to make adjustments of the transducer which updates the images continuously (DuBose, 2006). A psychomotor learning framework can be used to assist both the sonography students and teachers to identify the achieved stage of learning. Dawson's (1998) theoretical extension of Bloom's taxonomy can be extrapolated to create a framework for the competency assessment of sonography skills, which can guide the specific objectives of different learning opportunities. Educators can then more effectively facilitate the psychomotor skill of students during supervised practice and clinical placement.

In Australia, sonography education programs are delivered across a wide range of institutions including universities, professional organisations and private training schools. Predominantly, instructional design focuses on the cognitive and affective domain of learning, with less emphasis on the psychomotor domain. Cognitive skills for sonography are often taught using a didactic approach where information is conveyed to the students as a passive learning experience. This learning is then augmented through practical workshops which provide the students with a limited opportunity to observe and practise psychomotor skills prior to further skills development in the clinical setting. This approach probably arises from the convention of teaching institutions relying on qualified practitioners in the workplace to teach and develop sonographic psychomotor skills during supervised scanning practice in the clinical environment (Dresang, 2004). These practitioners often have limited understanding of instructional theory.

Before learning any psychomotor task, students are required to have background theory in the cognitive and affective domains (Dawson, 1998). In sonography there needs to be conceptualisation or cognitive learning where the broader context of the skill is appreciated by learning the anatomy, pathology, sonographic appearances and indications for the examination. Multimedia tools such as CDs or DVDs have been utilised as an alternative to practical workshops to augment transition from the commonly employed didactic teaching of the cognitive skills, to the psychomotor skill of practical scanning (Dresang, 2004).

Multimedia tools, compared to workshops, have the advantage of being flexible for access and can promote a student-centred approach to learning (Biggs, 1999), leading to improved learning outcomes (Clark \& Paivio, 1991; Laurillard, 2002,) through the promotion of deeper learning (Mayer, 2003; Schwan \& Riempp, 2004). The use of an multimedia tool as an interactive web based medium has also been identified as a positive adjunct for students learning gross anatomy in medicine and allied health (Bacro et al., 2000; Lewis, 2003; Salyers, 2007), and has been reported to be as effective as face to face teaching for the teaching of surface anatomy (Coffee \& Hillier, 2008). There appear to be no studies investigating the effectiveness of multimedia tools in developing the psychomotor skills required in medical sonography.

In this paper we report on a small pilot trial in which we investigated the performance and perceptions of medical sonography students who used a specifically designed multimedia tool to learn psychomotor skills of musculoskeletal ankle sonography. A competency assessment schema framed around Dawson's (1998) psychomotor categories was used to quantify changes in students' skill levels before and after the 
intervention. It was proposed that an alternative to the traditional instructional design, namely using a multimedia learning tool, and assessment of competency would assist educators to structure incremental teaching and assessment activities, enabling a more targeted approach to developing psychomotor skills. This may also assist clinical supervisors and academic teachers to work together to identify ways to tailor learning opportunities for the student, and relieve some of the burden of clinical teaching in the workplace.

\section{Method}

This pilot study trialed a DVD designed and developed specifically for medical sonography students learning the skills to perform a sonographic examination of the ankle. The study was approved by the Human Research Ethics Committee of the University of South Australia.

\section{DVD learning tool}

A multimedia DVD learning tool was developed using audiovisual recordings and still captures. It included anatomic images, and video clips demonstrating sonographic techniques of the ankle and foot, with corresponding real time ultrasound images and audiovisual demonstrations of pertinent surface anatomy landmark localisation (Figure 2). The DVD was formatted into eighteen sections, each addressing different anatomic structures of the ankle. These structures varied in their degree of difficulty. A menu was provided at the beginning of the DVD to facilitate access to each section. A DVD format was selected over network distribution across the University's learning management system, to ensure dependable access to the learning tool. In our experience, external students have reported variable success in accessing some online resources depending on the availability and speed of Internet services. The DVD addressed the first category of skill development ('observation') within the framework described by Dawson (1998). It provided the students with the opportunity to observe skills from video clips, and facilitated them to engage in 'trial' and 'repetition' independently following viewing.

\section{Investigation of learning outcomes}

Five postgraduate participants trialed the learning tool (DVD) to facilitate self directed development of sonography skills over a three month period between June and September 2009. The participants were either enrolled in or had recently graduated from a postgraduate sonography training program which was limited to teaching and assessing knowledge, but not skills in musculoskeletal sonography. Participants in the study were provided with additional learning and assessment activities to develop skills competencies that were not a mandatory part of the sonography program. All participants volunteered their involvement in the project, which did not affect their results in the courses/programs of study at the institution. Most skills demonstrated in the DVD were considered advanced skills not included at entry level practitioner training. A control group was not used for this study, because the learning environment for all participants was the workplace, subject to a variety of influences which are difficult to control. We planned instead to ask participants to self report on supervised and non-supervised learning experiences in the workplace to provide insight into the workplace learning environment for each individual. 


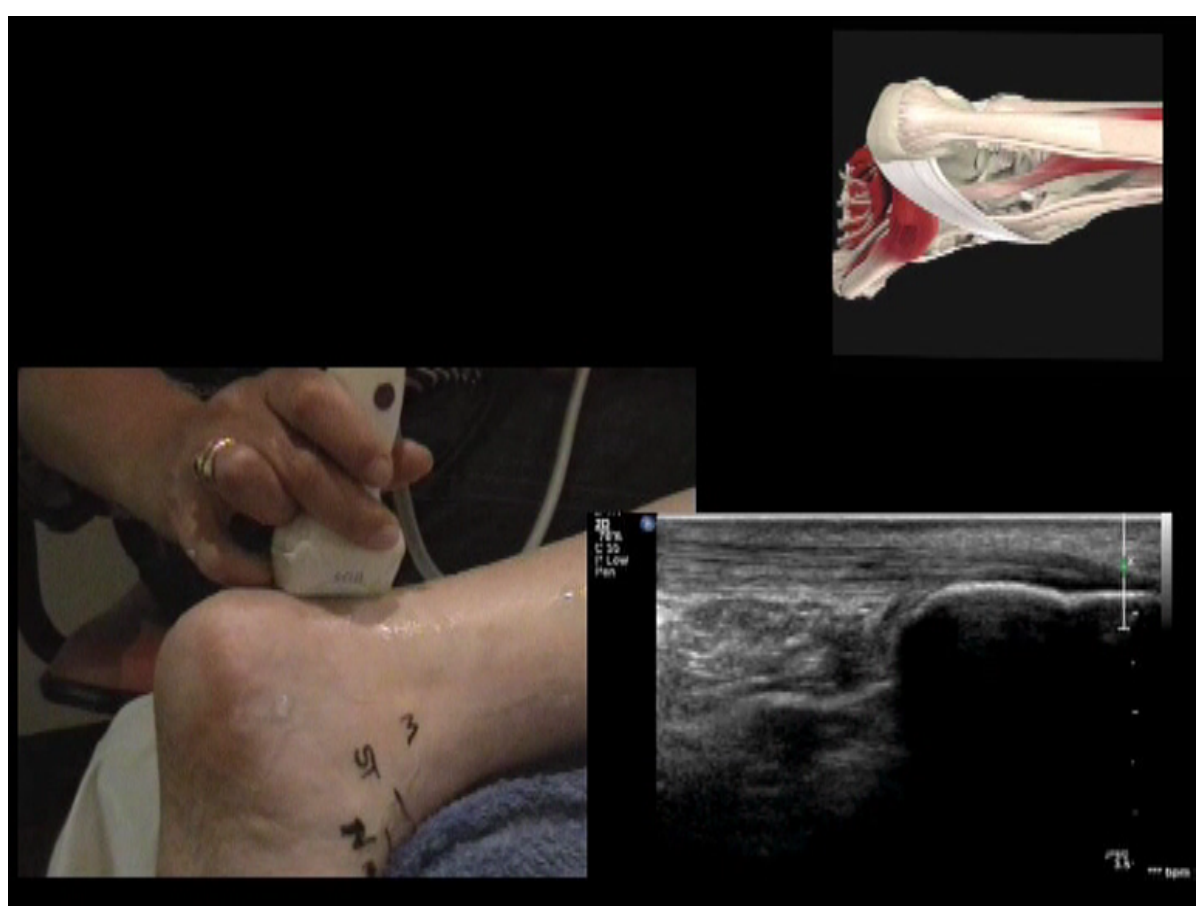

Figure 2: Screen capture of DVD

Participant competency was assessed on two occasions. Baseline competency testing occurred prior to the participant taking possession of the DVD, and post-intervention competency testing occurred three months after they had taken possession of the DVD. Each participant was provided with the self-directed learning tool (DVD) and given verbal instruction on both the learning objectives and the use of the tool immediately after the baseline competency testing. Participants were tested on their ability to demonstrate the eighteen anatomic structures of the ankle available on the DVD at the baseline and post-intervention competency testing. Competency was measured by an independent assessor who was an accredited sonographer experienced in musculoskeletal sonography. Participants were graded using three competency scores. The following competency scoring method, incorporating the psychomotor stages described by Dawson (1998), was used as a guide to determining the levels of competency (Table 1). The highest possible competency score for each anatomical structure was two (full competency). To assist in the participants' skill development feedback on performance was provided at the time of the baseline competency testing.

Following baseline testing, participants were given instruction in the use of the DVD for self directed learning. They were encouraged to augment the use of this tool with both non- supervised practice sessions and supervised real life clinical practice sessions. These practice sessions were performed using colleagues as real life models, or trying to identify these structures on real patients. Participants were asked to record the number of times they practised their skills in both supervised and unsupervised capacities in a log. Following the completion of the three month trial their opinions were canvassed via structured interviews regarding the design and use of the tool. 
Table 1: Competency scoring

\begin{tabular}{|c|c|c|l|}
\hline $\begin{array}{c}\text { Competency } \\
\text { score }\end{array}$ & $\begin{array}{c}\text { Dawson's } \\
\text { stage }\end{array}$ & $\begin{array}{c}\text { Competency } \\
\text { level }\end{array}$ & \multicolumn{1}{|c|}{ Description } \\
\hline 0 & Trial & No competency & $\begin{array}{l}\text { The participant could not localise, } \\
\text { recognise or demonstrate any part of the } \\
\text { anatomic structure using sonography }\end{array}$ \\
\hline 1 & Refinement & Partial competency & $\begin{array}{l}\text { The participant could localise, recognise } \\
\text { but only partially demonstrate the } \\
\text { anatomic structure using sonography }\end{array}$ \\
\hline 2 & Consolidation & Full competency & $\begin{array}{l}\text { The participant could localise, recognise } \\
\text { and demonstrate the complete anatomical } \\
\text { structure using sonography }\end{array}$ \\
\hline
\end{tabular}

\section{Results}

The five commencing participants completed the study.

\section{Grading of anatomic structures by level of difficulty}

Following baseline competency testing, the anatomic structures were graded into levels of difficulty by summing scores across all participants for each anatomic structure (Table 2).

Table 2: Grading of anatomic structures by level of difficulty

\begin{tabular}{|c|c|c|c|}
\hline \multicolumn{4}{|c|}{ Sum of baseline scores } \\
\hline $\begin{array}{l}9-10 \\
\text { (Easy) }\end{array}$ & $\begin{array}{c}6-8 \\
\text { (Moderately easy) }\end{array}$ & $\begin{array}{c}3-5 \\
\text { (Moderately difficult) }\end{array}$ & $\begin{array}{c}0-2 \\
\text { (Difficult) }\end{array}$ \\
\hline $\begin{array}{l}\text { Achilles } \\
\text { tendon }\end{array}$ & $\begin{array}{l}\text { Peroneus brevis } \\
\text { tendon }\end{array}$ & $\begin{array}{l}\text { Tibialis anterior tendon, Extensor } \\
\text { digitorum longus tendon, } \\
\text { Extensor hallucis longus tendon, } \\
\text { Anterior synovial recess, Anterior } \\
\text { talofibular ligament, Tibialis } \\
\text { posterior tendon, Flexor digitorum } \\
\text { longus tendon, Peroneus longus } \\
\text { tendon }\end{array}$ & $\begin{array}{l}\text { Superior retinaculum, } \\
\text { Calcaneofibular ligament, } \\
\text { Tibionavicular ligament, } \\
\text { Tibiocalcaneal ligament, } \\
\text { Tibiotalar ligament, Spring } \\
\text { ligament, Anterior tibiofibular } \\
\text { ligament, Flexor hallucis } \\
\text { longus tendon }\end{array}$ \\
\hline
\end{tabular}

\section{Participant competency results}

Participants' commencing competency level was graded by summing baseline testing scores across all structures. Two participants ( 1 and 2 ) were graded as low level (overall score 0-10), two (3 and 4) were graded as moderate level (overall score 11-25) and one (5) was graded as high level (overall score 26-36). At post-intervention competency testing, all participants improved their scores. The greatest overall improvement was made by Participant 2 whose score increased from 3 to 24 . The smallest overall improvement was made by Participants 1 and 5 whose scores increased from 3 to 8 and 31 to 36 , respectively.

\section{Competency results for anatomic structures}

The Achilles tendon was graded as easy, with only one participant failing full competency at baseline and all participants achieving full competency postintervention (Figure 3). The peroneus brevis tendon was graded as moderately easy 
with two participants meeting full competency at baseline, and only one participant failing full competency for this structure at post-intervention testing (Figure 4).

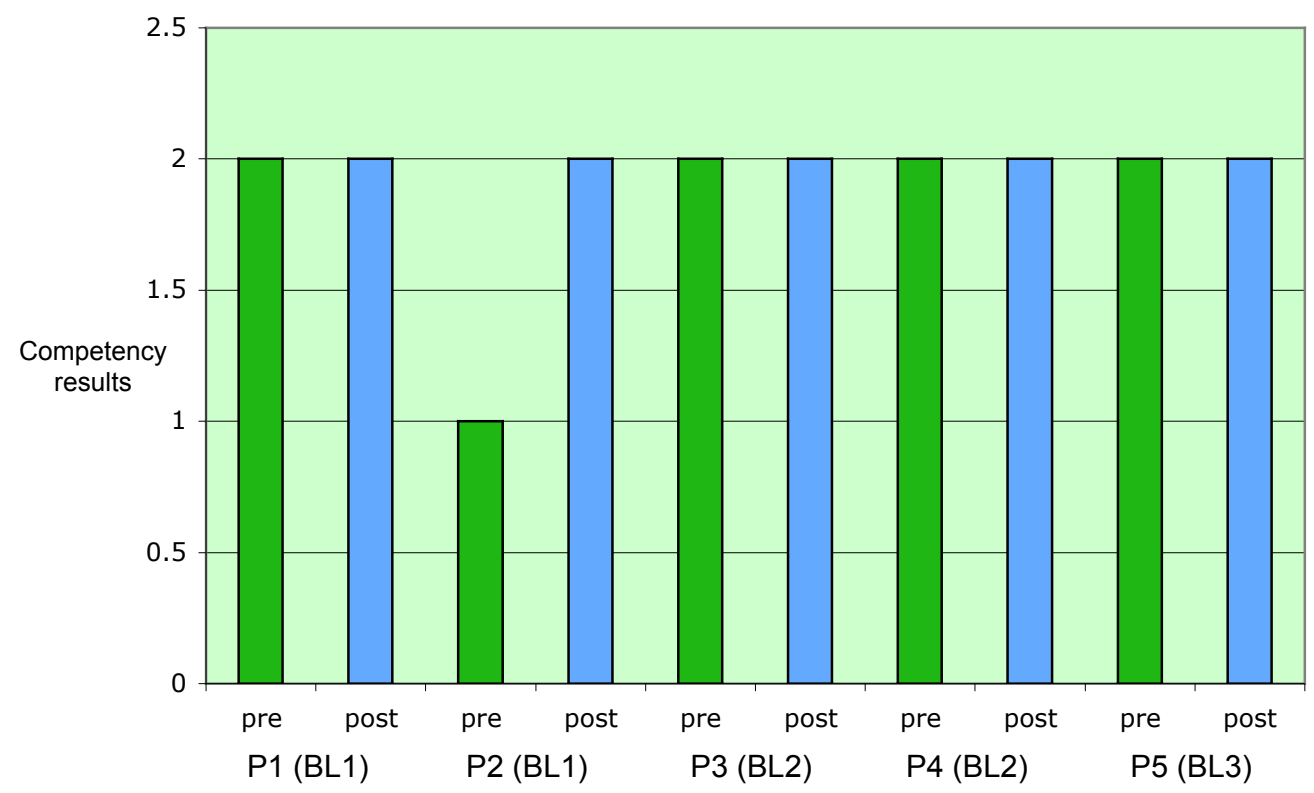

Figure 3: Achilles tendon: Competency test results, easy structure

Key: pre: baseline testing score; post: post-intervention testing score; P: participant BL 1: low level baseline competency 1; BL 2: moderate level baseline competency 2 BL 3: high level baseline competency 3

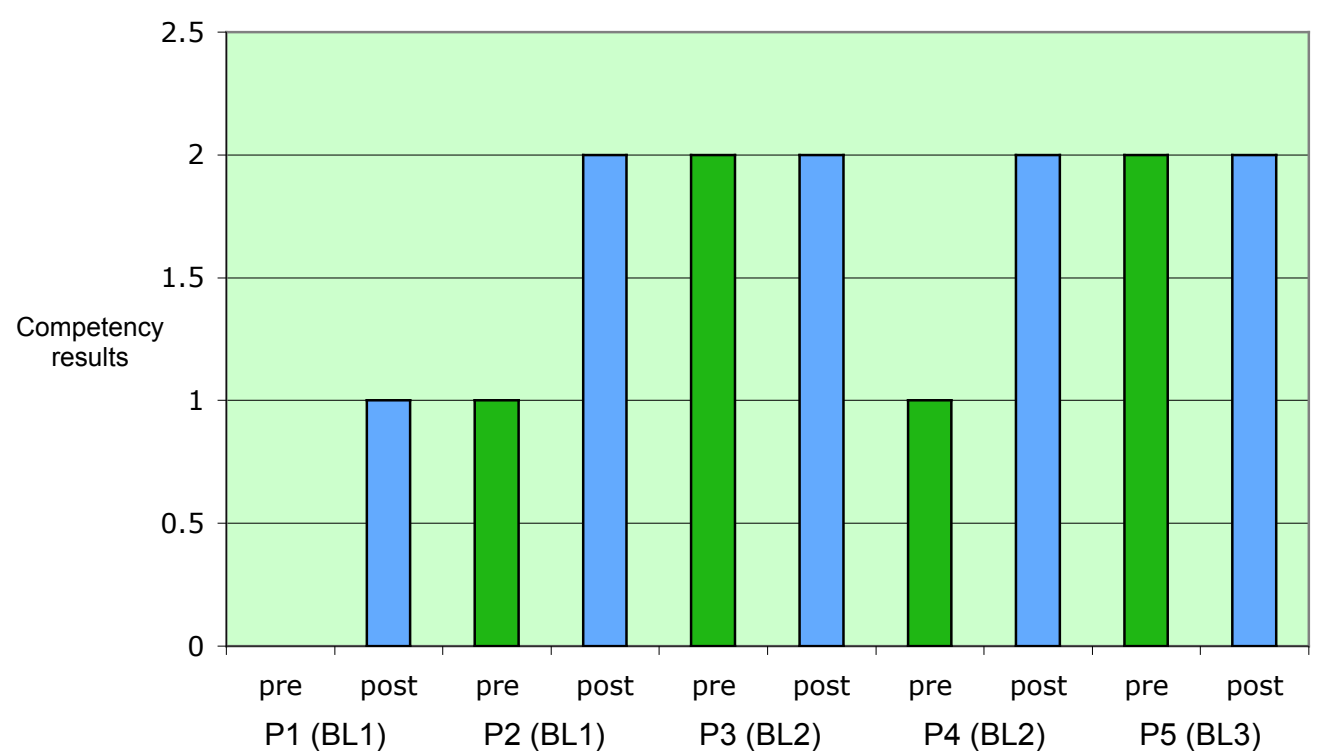

Figure 4: Peroneus tendon: Competency test results, moderately easy structure Key: As for Figure 3 above. 
Table 3 demonstrates changes in participant competency from baseline to postintervention for the eight moderately difficult structures. Participant 1 at low baseline competency, improved in three of the eight moderately difficult structures. Participant 2 commenced with low baseline scores, and showed improvement in seven of the eight structures, reaching full competency in six structures. Participant 3 with a moderate baseline level, demonstrated improvement and full competency in seven of eight structures. Participant 4 with moderate baseline competency, demonstrated full competence in seven of eight structures at post-intervention testing. Participant 5 at high baseline competency level, demonstrated full competency at baseline for seven of eight moderately difficult structures and attained full competency for every structure at post-intervention testing.

Table 3: Moderately difficult structures. Competency test results

\begin{tabular}{|c|c|c|c|c|c|c|c|c|c|c|}
\hline \multirow{3}{*}{ Anatomic structure } & \multicolumn{10}{|c|}{ Competency scores } \\
\hline & \multicolumn{2}{|c|}{ P1 (BL1) } & \multicolumn{2}{|c|}{ P2 (BL1) } & \multicolumn{2}{|c|}{ P3 (BL2) } & \multicolumn{2}{|c|}{ P4 (BL2) } & \multicolumn{2}{|c|}{ P5 (BL3) } \\
\hline & pre & post & pre & post & pre & post & pre & post & pre & post \\
\hline Tibialis anterior tendon & 1 & 1 & 0 & 2 & 2 & 2 & 0 & 2 & 2 & 2 \\
\hline $\begin{array}{l}\text { Extensor digitorum longus } \\
\text { tendon }\end{array}$ & 0 & 2 & 0 & 1 & 1 & 2 & 0 & 2 & 2 & 2 \\
\hline $\begin{array}{l}\text { Extensor hallucis longus } \\
\text { tendon }\end{array}$ & 0 & 1 & 0 & 2 & 2 & 2 & 0 & 1 & 2 & 2 \\
\hline Anterior synovial recess & 0 & 0 & 0 & 2 & 0 & 2 & 1 & 2 & 2 & 2 \\
\hline Anterior talofibular ligament & 0 & 1 & 0 & 2 & 0 & 0 & 1 & 2 & 2 & 2 \\
\hline Tibialis posterior tendon & 0 & 0 & 0 & 2 & 1 & 2 & 2 & 2 & 2 & 2 \\
\hline $\begin{array}{l}\text { Flexor digitorum longus } \\
\text { tendon }\end{array}$ & 0 & 0 & 0 & 2 & 0 & 2 & 1 & 2 & 2 & 2 \\
\hline Peroneus longus tendon & 0 & 0 & 1 & 1 & 1 & 2 & 1 & 2 & 1 & 2 \\
\hline
\end{tabular}

Key: pre: baseline testing score; post: post-intervention testing score; P: participant; BL 1: low level baseline competency 1; BL2: moderate level baseline competency 2; BL 3: high level baseline competency 3

All five participants improved their competency scores at post-intervention testing for moderately difficult structures (Figure 5).

The competency testing results for the eight difficult structures can be seen in Table 4 . Participant 1 at low baseline competency demonstrated no competency for difficult structures at baseline, improving for only one structure, but not reaching full competency. Participant 2 at low baseline competency, also failed to demonstrate competency for any difficult structures at baseline, but improved in three of eight structures at post-intervention testing, with three meeting full competency. Participant 3 at moderate baseline competency, also failed to demonstrate competency for difficult structures at baseline, but improved at post-intervention testing in five of eight structures, with full competency achieved for four structures. Participant 4 at moderate baseline competency demonstrated full competency for one out of eight difficult structures at baseline which was maintained post-intervention. There was improvement in three of the remaining seven structures with one meeting full competency post-intervention. Participant 5 at high baseline competency, achieved full competency in six out of eight difficult structures at baseline, and improved to achieve full competency for all structures at the post-intervention testing. 


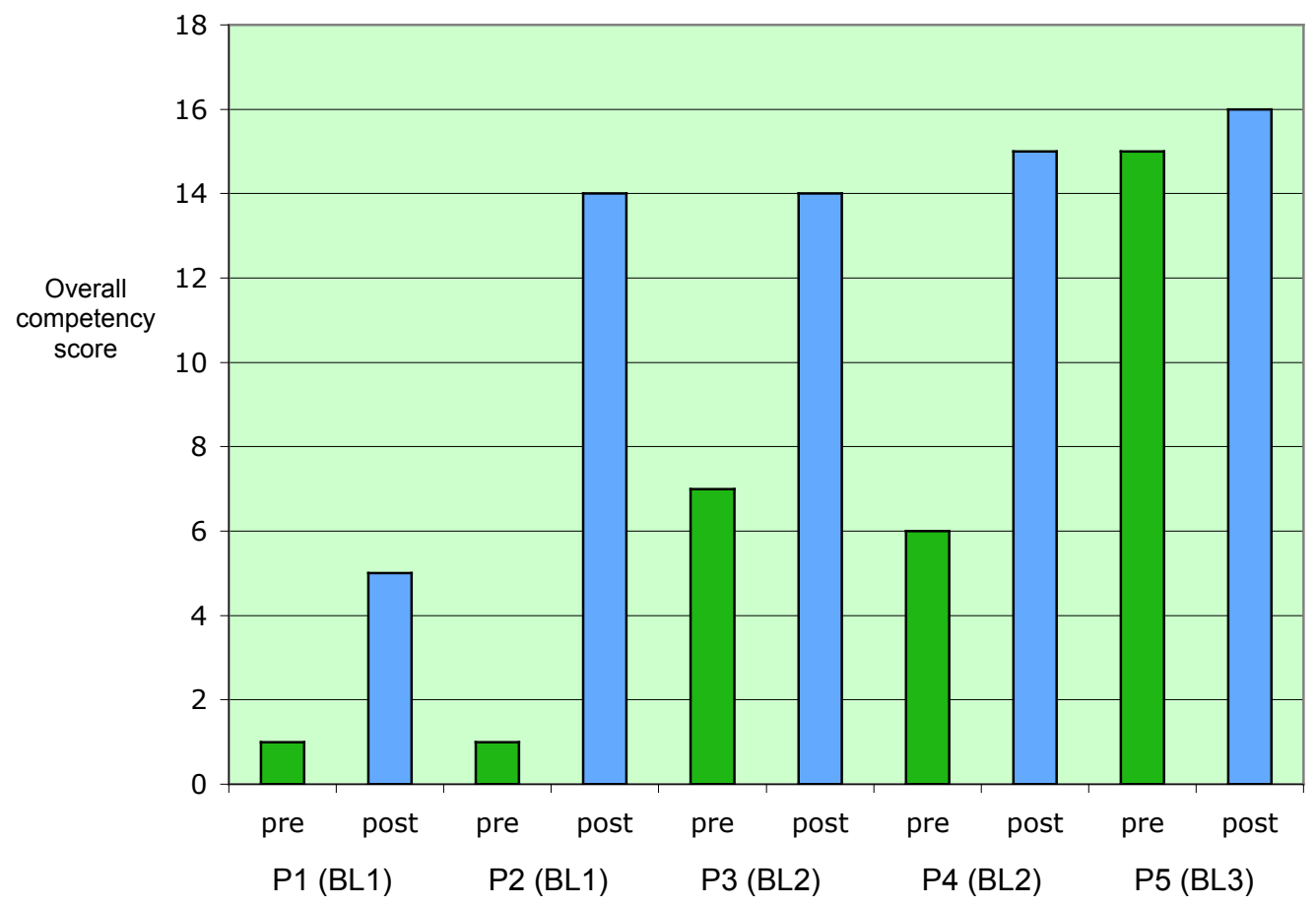

Figure 5: Overall competency testing scores, moderately difficult structures

Key: pre: baseline testing score; post: post-intervention testing score P: participant

BL 1: low level baseline competency 1

BL 2: moderate level baseline competency 2

BL 3: high level baseline competency 3.

Table 4: Difficult structures. Competency test results

\begin{tabular}{|l|c|c|c|c|c|c|c|c|c|c|}
\hline \multirow{3}{*}{\multicolumn{1}{c|}{ Anatomic structure }} & \multicolumn{9}{c|}{ Competency scores } \\
\cline { 2 - 14 } & P1 (BL1) & \multicolumn{1}{c|}{ P2 (BL1) } & P3 (BL2) & P4 (BL2) & \multicolumn{2}{c|}{ P5 (BL3) } \\
\cline { 2 - 12 } & pre & post & pre & post & pre & post & pre & post & pre & post \\
\hline Superior retinaculum & 0 & 0 & 0 & 2 & 0 & 2 & 0 & 0 & 2 & 2 \\
\hline Anterior tibiofibular ligament & 0 & 1 & 0 & 0 & 0 & 0 & 0 & 0 & 2 & 2 \\
\hline Calcaneofibular ligament & 0 & 0 & 0 & 2 & 0 & 0 & 0 & 2 & 2 & 2 \\
\hline Tibionavicular ligament & 0 & 0 & 0 & 0 & 0 & 1 & 0 & 0 & 2 & 2 \\
\hline Tibiocalcaneal ligament & 0 & 0 & 0 & 0 & 0 & 2 & 0 & 1 & 2 & 2 \\
\hline Tibiotalar ligament & 0 & 0 & 0 & 0 & 0 & 0 & 0 & 0 & 0 & 2 \\
\hline Spring ligament & 0 & 0 & 0 & 0 & 0 & 2 & 0 & 1 & 2 & 2 \\
\hline Flexor hallucis longus & 0 & 0 & 0 & 2 & 0 & 2 & 2 & 2 & 0 & 2 \\
\hline
\end{tabular}

Key: As for Figure 5 above.

Figures 5 and 6 demonstrate that more participants achieved full competency with moderately difficult structures compared to difficult structures. All participants retained at least the minimum demonstrated baseline competencies at the postintervention testing, consistent with the consolidation level described by Dawson (1998). Mastery was not tested in this pilot study as the testing situation was 
unchanged from baseline to post-intervention testing and the participant's ability to achieve competence in identifying these structures in all circumstances was not tested.

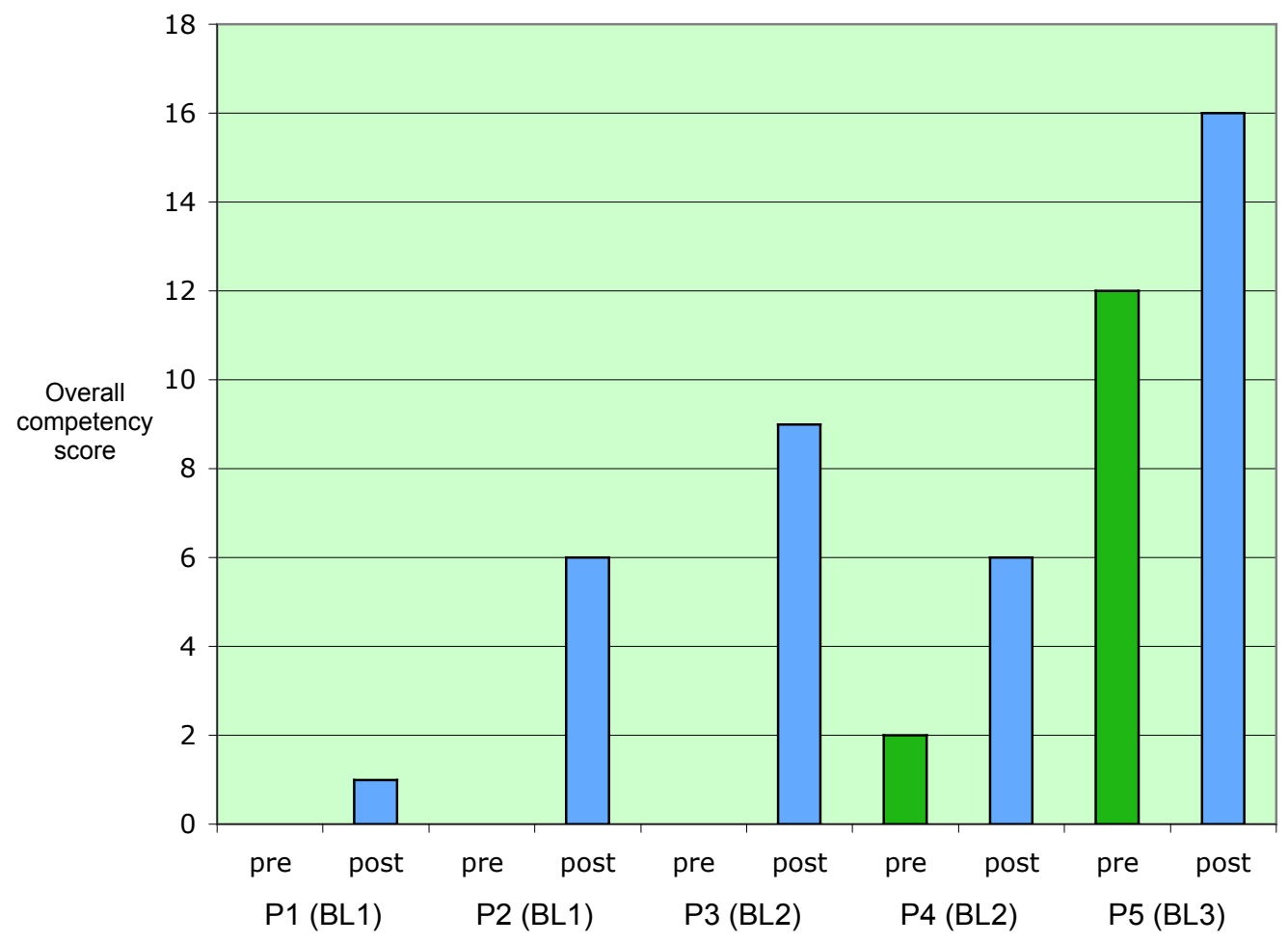

Figure 6: Overall competency testing, difficult structures

Key: pre: baseline testing score; post: post-intervention testing score; P: participant BL 1: low level baseline competency 1; BL 2: moderate level baseline competency 2 BL 3: high level baseline competency 3

Overall scores between baseline and post-intervention competency testing increased by $50 \%$. The highest post-intervention scores (8-10) were recorded for one easy structure (Achilles tendon), one moderately easy structure (peroneus brevis tendon), six moderately difficult structures (anterior synovial recess, tibialis anterior, extensor digitorum longus, extensor hallicus longus, tibialis posterior and flexor digitorum longus tendons) and one difficult structure (flexor hallicus longus tendon). The lowest post-intervention scores (0-3) were recorded for three difficult structures (anterior tibiofibular ligament, tibionavicular and spring ligaments). The tibio-talar and tibionavicular ligaments (Table 4) appear to have been the most difficult structures to attain full competence across all participants.

\section{Supervised and non-supervised practice sessions}

Participants were asked to maintain a log of the number of supervised and nonsupervised practice sessions they undertook for each structure. Participant 1 did not provide this, but reported she had limited non-supervised and no supervised practice 
over the three month period. Participant 2 indicated which structures she practised but not how many times she practised each structure. The supervised and non-supervised practice sessions are summarised in Table 5.

Table 5: Summary of supervised and non-supervised practice sessions.

\begin{tabular}{|c|c|c|c|c|c|c|}
\hline \multirow{2}{*}{$\begin{array}{l}\text { Level of } \\
\text { difficulty }\end{array}$} & \multirow{2}{*}{$\begin{array}{l}\text { Anatomical } \\
\text { structure }\end{array}$} & \multicolumn{5}{|c|}{ Participants } \\
\hline & & P1 (BL1) & P2 (BL1) & P3 (BL2) & P4 (BL2) & P5 (BL3) \\
\hline \multirow[t]{3}{*}{ Easy } & Achilles tendon & $\mathrm{S}=0$ & $\mathrm{~S}=0$ & $\mathrm{~S}=0$ & $\mathrm{~S}=0$ & $\mathrm{~S}=0$ \\
\hline & & $\mathrm{U}=$ limited & $U^{*}$ & $\mathrm{U}=8$ & $\mathrm{U}=12$ & $\mathrm{U}=62$ \\
\hline & & C 2-2 & C $1-2$ & C 2-2 & C 2-2 & C 2-2 \\
\hline \multirow{3}{*}{$\begin{array}{l}\text { Moderately } \\
\text { easy }\end{array}$} & Peroneus brevis & $S=0$, & $\mathrm{S}=0$ & $\mathrm{~S}=0$ & $\mathrm{~S}=5$ & $S=0$ \\
\hline & tendon & $\mathrm{U}=$ limited & $\mathrm{U}^{*}$ & $\mathrm{U}=4$ & $\mathrm{U}=12$ & $\mathrm{U}=47$ \\
\hline & & C $0-1$ & C $1-2$ & C 2-2 & C $1-2$ & C 2-2 \\
\hline \multirow{24}{*}{$\begin{array}{l}\text { Moderately } \\
\text { difficult }\end{array}$} & Tibialis anterior & $\mathrm{S}=0$ & $S=0$ & $\mathrm{~S}=0$ & $S=4$ & $S=0$ \\
\hline & tendon & $\mathrm{U}=$ limited & $\mathrm{U}^{*}$ & $\mathrm{U}=2$ & $\mathrm{U}=7$ & $\mathrm{U}=21$ \\
\hline & & C 1-1 & C $0-2$ & C 2-2 & C $0-2$ & C 2-2 \\
\hline & Extensor & $S=0$ & $\mathrm{~S}=0$ & $\mathrm{~S}=0$ & $\mathrm{~S}=4$ & $S=0$ \\
\hline & digitorum & $\mathrm{U}=$ limited & $\mathrm{U}^{*}$ & $\mathrm{U}=2$ & $\mathrm{U}=7$ & $\mathrm{U}=21$ \\
\hline & tendons (4) & C $0-2$ & C $0-1$ & C $1-2$ & C $0-2$ & C 2-2 \\
\hline & Extensor & $\mathrm{S}=0$ & $\mathrm{~S}=0$ & $\mathrm{~S}=0$ & $\mathrm{~S}=4$ & $\mathrm{~S}=0$ \\
\hline & hallucis longus & $\mathrm{U}=$ limited & $\mathrm{U}^{*}$ & $\mathrm{U}=2$ & $\mathrm{U}=7$ & $\mathrm{U}=21$ \\
\hline & & C 0-1 & C $0-2$ & C 2-2 & C $0-1$ & C $2-2$ \\
\hline & Anterior & $\mathrm{S}=0$ & $\mathrm{~S}=0$ & $S=0$ & $\mathrm{~S}=10$ & $S=0$ \\
\hline & synovial recess & $\mathrm{U}=$ limited & $\mathrm{U}^{*}$ & $\mathrm{U}=6$ & $\mathrm{U}=15$ & $\mathrm{U}=21$ \\
\hline & & C $0-0$ & C $0-2$ & $\mathrm{C} 0-2$ & C $1-2$ & C $2-2$ \\
\hline & Anterior & $S=0$ & $\mathrm{~S}=0$ & $S=3$ & $S=5$ & $\mathrm{~S}=0$ \\
\hline & talofibular & $\mathrm{U}=$ limited & $U^{*}$ & $\mathrm{U}=0$ & $\mathrm{U}=10$ & $\mathrm{U}=31$ \\
\hline & ligament & C $0-1$ & C $0-2$ & C $0-0$ & C $1-2$ & C 2-2 \\
\hline & Tibialis & $\mathrm{S}=0$ & $\mathrm{~S}=0$ & $\mathrm{~S}=0$ & $S=7$ & $S=0$ \\
\hline & posterior & $\mathrm{U}=$ limited & $\mathrm{U}^{*}$ & $\mathrm{U}=3$ & $\mathrm{U}=10$ & $\mathrm{U}=41$ \\
\hline & tendon & C $0-0$ & C $0-0$ & C $1-2$ & C 2-2 & C 2-2 \\
\hline & Flexor & $S=0$ & $\mathrm{~S}=0$ & $\mathrm{~S}=0$ & $\mathrm{~S}=7$ & $S=0$ \\
\hline & digitorum & $\mathrm{U}=$ limited & $\mathrm{U}^{*}$ & $\mathrm{U}=3$ & $\mathrm{U}=10$ & $\mathrm{U}=41$ \\
\hline & longus tendon & C $0-0$ & C $0-2$ & $\mathrm{C} 0-2$ & C $1-2$ & C $2-2$ \\
\hline & Peroneus & $\mathrm{S}=0$ & $\mathrm{~S}=0$ & $\mathrm{~S}=0$ & $\mathrm{~S}=5$ & $\mathrm{~S}=0$ \\
\hline & longus tendon & $\mathrm{U}=$ limited & $\mathrm{U}^{*}$ & $\mathrm{U}=4$ & $\mathrm{U}=12$ & $\mathrm{U}=47$ \\
\hline & & C $0-0$ & C $1-1$ & C $1-2$ & C $1-2$ & C $1-2$ \\
\hline \multirow{18}{*}{ Difficult } & Calcaneofibular & $\mathrm{S}=0$ & $\mathrm{~S}=0$ & $S=3$ & $\mathrm{~S}=5$ & $\mathrm{~S}=0$ \\
\hline & ligament & $\mathrm{U}=$ limited & $\mathrm{U}^{*}$ & $\mathrm{U}=0$ & $\mathrm{U}=11$ & $\mathrm{U}=31$ \\
\hline & & C $0-0$ & C $0-02$ & C $0-0$ & C $0-2$ & C 2-2 \\
\hline & Tibionavicular & $\mathrm{S}=0$ & $\mathrm{~S}=0$ & $\mathrm{~S}=2$ & $S=3$ & $S=1$ \\
\hline & ligament & $\mathrm{U}=$ limited & $\mathrm{U}=0$ & $\mathrm{U}=0$ & $\mathrm{U}=5$ & $\mathrm{U}=15$ \\
\hline & & C $0-0$ & C $0-0$ & $\mathrm{C} 0-0$ & $\mathrm{C} 0-0$ & C $0-2$ \\
\hline & Tibiocalcaneal & $\mathrm{S}=0$ & $\mathrm{~S}=0$ & $\mathrm{~S}=2$ & $S=3$ & $S=1$ \\
\hline & ligament & $\mathrm{U}=$ limited & $\mathrm{U}=0$ & $\mathrm{U}=0$ & $\mathrm{U}=3$ & $\mathrm{U}=15$ \\
\hline & & C $0-0$ & C $0-0$ & C $0-2$ & C 0-1 & C 2-2 \\
\hline & Tibiotalar & $S=0$ & $\mathrm{~S}=0$ & $S=2$ & $S=3$ & $\mathrm{~S}=1$ \\
\hline & ligament & U =limited & $\mathrm{U}=0$ & $\mathrm{U}=0$ & $\mathrm{U}=3$ & $\mathrm{U}=15$ \\
\hline & & C $0-0$ & C $0-0$ & C $0-0$ & C $0-0$ & C $0-2$ \\
\hline & Spring ligament & $S=0$ & $S=0$ & $S=2$ & $S=3$ & $S=1$ \\
\hline & & $\mathrm{U}=$ limited & $\mathrm{U}=0$ & $\mathrm{U}=0$ & $\mathrm{U}=2$ & $\mathrm{U}=12$ \\
\hline & & C $0-0$ & C $0-0$ & $\mathrm{C} 0-2$ & C $0-1$ & C $2-2$ \\
\hline & Superior & $\mathrm{S}=0$ & $\mathrm{~S}=0$ & $\mathrm{~S}=0$ & $S=2$ & $\mathrm{~S}=0$ \\
\hline & retinaculum & $\mathrm{U}=$ limited & $U^{*}$ & $\mathrm{U}=4$ & $\mathrm{U}=2$ & $\mathrm{U}=21$ \\
\hline & & C $0-0$ & C $0-2$ & C $0-2$ & C $0-0$ & C 2-2 \\
\hline
\end{tabular}




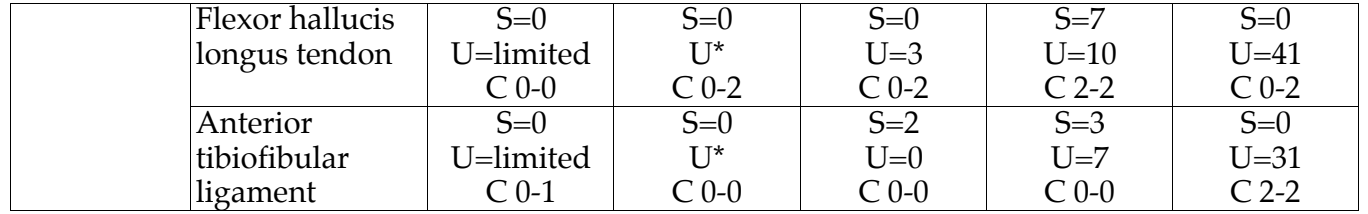

Key: P: Participant; S: supervised training sessions; U: non-supervised training sessions;

*: indicates training sessions occurred; $C$ : change in competency from baseline test to post-intervention test; BL 1: low level baseline competency 1; BL2: moderate level baseline competency 2; BL 3: high level baseline competency 3.

Participants appeared to engage in non-supervised sessions more frequently than supervised sessions. Participants were able to demonstrate an improvement in their competency scores with supervised or non-supervised practice sessions or a combination of both across all ranges of difficulty.

\section{Interviews}

Following the post-intervention competency testing participants were interviewed and asked a series of questions regarding their opinions and experiences of using the DVD learning tool. The questions explored three broad themes; their use behaviour, the perceived benefits and limitations of using the learning tool, and a comparison between self paced and face to face tuition.

All participants reported having used the DVD prior to practising in clinic and reported the DVD format was easily navigable and in a familiar format. They reported the surface anatomy section particularly useful for revision in localising anatomical landmarks. All participants commented they found the audiovisual format beneficial for their learning. One participant commented:

I have said before I am a very visual person it is better to look at stuff... I see it essential to go with readings and other materials. I think it is an essential part because ultrasound is such a practical modality and if you don't have a visual thing in your head to know if you are on the right track or not it makes it a bit hard. Yes I think it is important (Participant 2).

Participants were asked to compare this method of instructional delivery with the face to face teaching workshop and all preferred the face to face teaching workshop because of the limitations of the DVD when seeking clarification. Two participants commented that:

[the DVD was] an advantage for beginning practitioners to get the ground work before going to supervised practice (Participant 5).

They go hand in hand, so you need to do both... I think (Participant 2).

Participants stated they gained more learning from face to face teaching because of the immediate feedback they received on their techniques, and the opportunity to receive immediate answers to their questions. However one participant cited the DVD had particular benefits because:

If you forget something you can go back and look at it whereas you only retain some part maybe $30 \%$ of the workshop but [they] have to view it a few times (Participant 4 ). 


\section{Discussion}

The DVD was developed as an instructional tool to assist students' transition from the teaching and learning of cognitive skills, to the development of psychomotor skills of practical sonography. This is a particular challenge in our institution, because almost all of our sonography teaching is, by necessity, provided through external delivery. In this format minimal support can be provided for the practical skills training. For this reason we require learning tools that can provide effective, self directed learning opportunities for our students, to assist them in the development of practical clinical skills.

The multimedia DVD provided participants in our pilot study with the opportunity to observe sonography techniques being performed to supplement the necessary anatomical knowledge. This enabled them to commence the trial and repetition psychomotor learning (Dawson, 1998) sequence of scanning each structure. Compared to other medical imaging modalities, sonography skills can be developed by practising on consenting volunteers with low risk of inducing biological effects. This means that there is opportunity for students to engage safely in Dawson's (1998) learning loop without supervision. In this way a participant could potentially achieve a beginning level of competence with repeated scanning practice. Our participants indicated that they used the DVD to observe technique, and to compare the images scanned with those included for each structure on the DVD, facilitating their ability to reflect on and adapt their performance to achieve successful reproduction of the sonographic images for each structure.

The small number of participants, and the absence of a control group in this study limits our ability to generalise our findings, or to make assessments of the effectiveness of our learning tool. Recruitment of participants was difficult due to the wide geographic distribution of students, and their unwillingness to travel to the University on two occasions for competence testing. This study aimed to explore ways to expand the student learning experience, however the external mode of teaching, while attractive for its flexible delivery, hindered student recruitment. Despite its limitations the study did provide some insight into the perceptions and acceptance of the multimedia tool by the students. Future research could include a larger trial, after integrating the tool and competency testing instrument into the formal teaching program.

Despite the small sample size, it did include individual participants across a range of competency levels; two participants had very low competence; two had moderate competence, and one high competence. The greatest improvements in scoring between pre- and post-intervention competency testing were seen in the participants with moderate baseline competence, and one participant with low baseline competence. The student with low baseline competence who had limited improvement also indicated no supervised and low levels of unsupervised practice sessions.

All participants in this study improved, with a range of supervised and unsupervised training, and baseline competency levels. It is likely that the DVD had a positive effect on the acquisition of competency in musculoskeletal sonography of specific ankle structures, although we cannot exclude other influences on the improvements in competency. It was not possible to control for the training experiences the participants received in the three month intervention period. The baseline competency testing session provided an extraneous learning experience for the students. 
Interestingly, most participants improved with minimal supervised training. Participants in this study were able to attain full competence in demonstrating several anatomical structures of the ankle sonographically after a period of practice using the DVD as a learning tool without supervision (Table 2). In contrast, one participant was supervised when practising sonography skills for seven of the structures (Participant 3), but was only able to achieve full competence in two of these at the post-intervention testing. Participant 4 had the most balanced combination of supervised and unsupervised practice, with more supervision than the other participants. This participant's competency score overall was 7 out of 18 at post-intervention testing, a comparable result to participants for whom no supervision had occurred.

It would appear that participants' unsupervised practice may be an effective mechanism for skills development and improvement. This is no surprise to those teaching the psychomotor skills in the allied health sciences. However the implication for teaching in this cohort may mean that educators can reasonably expect students to practise unsupervised and gain a level of competency in scanning the easy, moderately easy and several of the moderately difficult structures of the ankle using such a learning tool. The number of unsupervised sessions needed to achieve this will vary for each participant and is a topic for further study.

Analysis of the results of post competency testing reveals the structures that participants had the least and most difficulty in demonstrating. The tibio-talar and tibio-navicular ligaments were demonstrated by the fewest participants, suggesting these structures have the greatest degree of difficulty. Conversely the Achilles and the peroneus brevis tendons could be considered to be more easily identified with most participants attaining competency. The Achilles tendon was the only structure which could be competently demonstrated at baseline testing by all participants. This structure is large, easily identifiable and commonly imaged with sonography. It is no surprise that participants demonstrated this structure well. The anterior tibio-fibular ligament recorded the lowest scores and full competency was not achieved after DVD intervention.

Other musculoskeletal structures of the ankle that rated low scores were the ligamentous structures of the medial ankle (spring, tibiocalcaneal, tibionavicular, and tibiotalar ligaments). This is also not surprising, as most sonographers find these structures difficult to image. Improvement of skills in demonstrating these structures occurred for participants with moderate or high baseline skill level. A different instructional design for teaching may be more suitable to develop skills in demonstrating these structures.

Participants thought the DVD was a useful learning tool and indicated that they utilised it in their learning, but were reluctant for it to replace face to face teaching sessions or clinical instruction, mostly because they valued feedback and guidance on their performance which the DVD could not provide. Feedback is fundamental to good clinical teaching (Branch \& Paranjape, 2002), clinically focused teaching provides a forum for delivering feedback, and also provides students experience in other domains of sonography training including communication, pathology recognition and clinical decision making, that the DVD did not address. There is however potential to enhance teaching with the DVD, by integrating its use with face to face teaching and teaching in the clinical environment within a structured learning framework. 


\section{Conclusion}

This innovative learning tool has implications for the instructional design of campus based intensive workshops and supervised clinical practice. These psychomotor skills are usually introduced through workshops where the student may or may not be offered hands on tuition, or through observation in the clinical setting. The effective use of teaching time in both settings is influenced by the number of participants, competing clinical loads, or variation in baseline skills across students. Providing students with a learning tool like the one used in this pilot study, prior to attendance at a workshop or real clinical demonstration could assist students to prepare and highlight specific areas for them to work on. The instruction provided during workshops could then be targeted at an assumed standardised skill level, and dedicated attention paid to the teaching and supervised practice of skills in identifying the moderately difficult and difficult structures. The preliminary assessment of competency could be used to determine the level of student skill, and direct focused teaching to that level. This approach has implications for improving teaching efficiency, which is especially important in the clinical setting where teaching is often secondary to the demands of patient service delivery. This alternative to traditional instructional design may be applicable and transferable to other areas of psychomotor skill training in the health setting.

This study, although limited by small sample size, suggests there is a positive relationship between use of the DVD, unsupervised practice and competency development. The results are promising, and larger studies with control groups should be performed to test the effect of the multimedia DVD learning tool on competency outcomes. Further investigation also needs to be undertaken to determine the role this and other learning tools such as supervised practice have within learning frameworks for developing specific psychomotor clinical skills.

\section{References}

Bacro, T., Gilbertson, B. \& Coultas, J. (2000). Web delivery of anatomy video clips using a CDROM. The Anatomical Record, 261(2), 78-82. http: / / dx.doi.org/10.1002/(SICI)10970185(20000415)261:2<78::AID-AR7>3.0.CO;2-E

Biggs, J. (1999). What the student does: Teaching for enhanced learning. Higher Education Research and Development, 18, 57-75. http:/ / dx.doi.org/10.1080/0729436990180105

Bloom, B. S. (1956). Taxonomy of Educational Objectives. Handbook I: The Cognitive Domain. New York: David McKay Co Inc.

Branch, W. \& Paranjape, A. (2002). Feedback and reflection: Teaching methods for clinical settings. Academic Medicine, 77(12), 1185-1188. http: / / www.uthscsa.edu/gme/documents/FeedbackandReflection.pdf

Clark, J. M. \& Paivio, A. (1991). Dual coding theory and education. Educational Psychology Review, 3(3), 149-170. http:/ / www.hum.uu.nl/ medewerkers/bergh102/Language\%20Education/ Artikelen/Clark\%26Paivio.pdf

Coffee, J. \& Hillier, S. (2008). Teaching pre-cursor clinical skills using an online audio-visual tool: An evaluation using student responses. MERLOT Journal of Online Learning and Teaching, 4(4), 469-476. http: / / jolt.merlot.org/vol4no4/coffee_1208.pdf

Dave, R. H. (1975). Psychomotor levels. In R. J. Armstrong (Ed), Developing and writing behavioural objectives. Tucson, Arizona: Educational Innovators Press. 
Dawson, W. R. (1998). Extensions to Bloom's taxonomy of educational objectives. Sydney, Australia: Putney Publishing.

Dresang, L. T., Rodney, W. M. \& Dees, J. (2004). Teaching prenatal ultrasound to family medicine residents. Family Medicine, 36(2), 98-107. http: / / www.stfm.org/fmhub/fm2004/February/Lee98.pdf

DuBose, T. J. (2006). Sonography, what is it? Words are golden. Advance for Imaging \& Radiation Therapy Professional, 19(14), 12. http:/ / www.newtech-medical.com/articles/SonographyWhat-is-it-Words-are-Golden.html

Harrow, A. (1972). A taxonomy of psychomotor domain: A guide for developing behavioral objectives. New York: David McKay.

KPMG (2009). Health workforce in Australia and factors for current shortages. Australia: National Health Workforce Taskforce.

http: / / www.ahwo.gov.au / documents / NHWT / The\%20health\%20workforce\%20in $\% 20$ Aust ralia $\% 20$ and $\% 20$ factors $\% 20$ influencing $\% 20$ current $\% 20$ shortages.pdf

Laurillard, D. (2002). Rethinking university teaching: A conversational framework for the effective use of learning technologies. London: Routledge.

Lewis, M. J. (2003). Computer assisted learning for teaching anatomy and physiology in subjects allied to medicine. Medical Teacher, 25(2), 204-207.

http: / / informahealthcare.com/doi/abs/10.1080/0000000000000000000a

Mayer, R. E. (2003). The promise of multimedia learning: using the same instructional design methods across different media. Learning and Instruction, 13, 125-139. http: / / dx.doi.org/10.1016/S0959-4752(02)00016-6 [also at http: / / sam.arts.unsw.edu.au/media/File/ MayerMediaMethod03.pdf]

Salyers, V. L. (2007). Teaching psychomotor skills to beginning nursing students using a webenhanced approach: A quasi-experimental study. International Journal of Nursing Education Scholarship, 4(1), article 11. http:/ / dx.doi.org/10.2202/1548-923X.1373 [also at http: / / works.bepress.com / cgi / viewcontent.cgi?article=1007\&context=dr_vincent_salyers]

Schwan, S. \& Riempp, R. (2004). The cognitive benefits of interactive videos: Learning to tie nautical knots. Learning and Instruction, 14, 293-305.

http: / / dx.doi.org/10.1016/j.learninstruc.2004.06.005

Simpson, E. J. (1972). The classification of educational objectives in the psychomotor domain. Washington, DC: Gryphon House.

Authors: Dr Kerry Thoirs, International Centre for Allied Health Evidence and School of Health Sciences, University of South Australia, GPO Box 2471, Adelaide, South Australia 5000.Email: kerry.thoirs@unisa.edu.au

Jane Coffee, International Centre for Allied Health Evidence and School of Health Sciences, University of South Australia, GPO Box 2471, Adelaide, South Australia 5000. Email: jane.coffee@unisa.edu.au

Please cite as: Thoirs, K. \& Coffee, J. (2012). Developing the clinical psychomotor skills of musculoskeletal sonography using an multimedia DVD: A pilot study. Australasian Journal of Educational Technology, 28(4), 703-718.

http: / / www.ascilite.org.au/ajet/ajet28/thoirs.html 